\title{
A Tale of Two Perspectives: How Psychology and Neuroscience Contribute to Understanding Personhood
}

\author{
ERIN I. SMITH \\ California Baptist University, USA \\ esmith@calbaptist.edu \\ ORCID: 0000-0003-2388-4024
}

\begin{abstract}
Empirical science, such as psychology and neuroscience, employ diverse methods to develop data driven models and explanations for complex phenomena. In research on the self, differences in these methods produce different depictions of persons. Research in developmental psychology highlights the role of intuitive beliefs, such as psychological essentialism and intuitive dualism, in individuals' singular, cohesive, and stable sense of self. On the other hand, research in neuroscience highlights the de-centralized, distributed, multitudes of neural networks in competition making selves, with arguments around whether the interpretation of these data imply that the self is somehow fundamental and special to human functioning. In this paper, I explore these discrepant pictures of the self to advance understanding about personhood. Specifically, I suggest that these divergent pictures of self from psychology and neuroscience have the potential to inform philosophical and theological discussions around personhood by anchoring models of persons in empirical data. Likewise, I explore the opportunity for philosophy and theology to inform and enhance scientific research on the self by critiquing scientific bias and construct development as well as highlighting potential limits in understanding selves with empirical models.
\end{abstract}

Keywords: Self; identity; psychological essentialism; intuitive dualism; neuroscience; philosophy. 


\section{Introduction}

In a recent conversation between children, an 8-year-old laughed when a 5-year-old said she did not know what the word "personality" meant. The laughter stopped when the elder child was asked to define it for the younger. It seems the older child had an intuitive sense of personality but lacked the ability to define it. Psychologists, neuroscientists, philosophers, and theologians take turns being the older and the younger sibling in equivalent engagements around the topic of personhood. Some difficulties presented in examinations of personhood stem from complexities inherent to examining the thing we are; empirical investigations of personhood are influenced in important ways by the starting point of the observation. In this paper, I highlight how different representations of persons emerge from different methods of investigation. First, I detail psychological research describing intuitive beliefs about selves as singular, stable, and coherent, beliefs rooted in typical developmental constraints. Following, I present neuroscientific views of selves as decentralized, competing multitudes. I conclude by considering how discrepancies between these perspectives present possibilities for advancing ideas about personhood, inviting engagement between empirical sciences (i.e., psychology and neuroscience) and the humanities (i.e., philosophy and theology). I argue that multiple disciplines, methods, and perspectives can enrich and extend understanding about personhood.

\section{Psychological Perspectives}

Psychologists do not often talk about personhood; rather, the focus is on a complex, interrelated network of ideas around identity and self. Talaifar and Swann (2018) characterize "the self as a multifaceted, dynamic, and temporally continuous set of mental representations” (p. 2), an interchangeable term with identity. In this sense, self is the thing that we, as selves, use to answer the fundamental question about what it means "to be me," to be a person. Developmental psychology has documented that 
a sense of stable identity is present early in childhood and persists-even if implicitly-throughout the lifespan (Gelman 2003). This intuition, driven by psychological essentialism and intuitive dualism, is central to shaping beliefs about the nature of personhood.

\subsection{Psychological Essentialism}

Psychological essentialism refers to the tendency of individuals to attribute internal, non-obvious, unchanging attributes as the underlying cause of an entity's true nature or identity (Gelman 2003). Essences need not be conceptually coherent or clearly articulated; children as young as 3 -years-old display psychological essentialism, reasoning that the inside of a living thing is what determines the outside of the thing (Gelman and Wellman 1991). By the age of 4 , children consistently reason that identity conferring internal essences are unchanged by physical changes. A horse is a horse because its internal, unchanging "horse essence"; starting in early childhood it is understood intuitively that painting it to look like a zebra does not change its essence or its identity (Keil 1992).

Psychological essentialism emerges early across diverse cultures, possibly due to a more fundamental bias in human cognition (Cimpian and Salomon 2014). However, the use of psychological essentialism as an identity-conferring system is not immune to experience. For instance, Newman and Keil (2008) find that as children age, explanations for essences shifts from a view that essences are in a single place (i.e., the brain) to a view that they are distributed within an entity. This developmental shift moves children closer to adults' essentialist-like reasoning about DNA. Moreover, individuals' tendencies to essentialize category members also differs according to features of cultural input and language (Heyman and Giles 2006), suggesting that human cognitive defaults are not deterministic.

Importantly, psychological essentialism is not restricted to categories (e.g., horses, humans), but extends to individual identities. In a series of studies, Johnson (1990) gave 5- to 11-year-old children scenarios in which the child's own brain replaced a pig's brain, or the child's own brain, mouth, heart, or face replaced/was replaced by the brain, mouth, heart, 
or face of a baby, similarly aged child, or older child. These experimental manipulations allowed Johnson to examine children's beliefs about the role of different internal (possibly essential) and external body parts in conferring identity. Across the studies, most children identified the brain as more fundamental than other body parts in conferring identity. When asked "who are you?", children answered that they were whatever entity housed their brain. This suggests that children have some understanding of the role of the brain in identity (compared to the heart, mouth, and face). Notably, however, this research did not assess children's conceptions of minds in identity. Although understanding the connectedness between brain and mind is increasingly common (Riekki, Linderman, and Lipsanen 2013), it is still exceptional given evidence indicating that the human default position is one of separation of the physical body/brain and mind (e.g., Bloom, 2004). Other research probing children's identity intuitions indicated that it is more believable to duplicate physical bodies without minds and that the duplication of physical parts does not also bring properties of the mind (e.g., memories; Hood et al. 2012), properties associated with identity.

The ease and frequency of explanations consistent with psychological essentialism has implications that extend beyond the direct question of how we understand identity and personhood. For example, one derivative of psychological essentialism important in a psychological view of personhood is intuitive dualism (Forstmann and Burgmer 2015).

\subsection{Intuitive Dualism}

Intuitive dualism refers to an untaught belief that minds and bodies are distinct and separable (Bloom, 2004). Explorations of intuitive dualism often focus on its connection to persistent and widespread beliefs about an afterlife (e.g., Riekki, et al. 2013, but see also Hodge 2008). For the present argument, it is important to articulate the content of the part-of-self (or, essence) that is believed to continue after death.

There is a rich body of research in psychology documenting the developmental trajectories of beliefs about minds. Since the first use of the term theory of mind (Premack and Woodruff 1978), research on children's 
developing understanding that other people have minds-thoughts, beliefs, motivations-that are different from their own and that may be false has pervaded numerous domains of psychological research (Beaudoin, Leblanc, Gagner, and Beauchamp 2019). In many religious and cultural contexts, these beliefs may be further refined and differentiated with concepts of the soul, spirit, or vitality/life force (Bering 2006; Richert and Harris 2008; Roazzi, Nyhof, and Johnson 2013).

The way individuals conceptualize physical and mental life has important implications. For example, Forstmann, Burgmer, and Mussweiler (2012) demonstrate that dualistic (versus materialistic) views of human persons influence health behaviors. Even among mental health care professionals, differing views about disorders as being in the body (e.g., schizophrenia) versus being in the mind (e.g., adjustment disorders) led to different conclusions about the effectiveness of biological/medical interventions compared to psychotherapeutic interventions (Ahn, Proctor, and Flanagan 2009). Moreover, it seems that even if individuals adopt explicitly monistic views of personhood, dualistic cognitive intuitions can still emerge in thinking and behavior (Forstmann and Burgmer 2015), suggesting that these intuitions persist across the lifespan. Research suggests that when individuals reason about separate bodies and minds (and/ or souls), essential elements of identity are wrapped up with the immaterial constructs, a finding that holds even across cultures that differ in important ways in their explicit and cultural treatment of these ideas (e.g., Cohen, Burdett, Knight, and Barrett 2011; Chudek et al. 2018).

\subsection{Intuition and Experience}

Although intuitions around the essential nature of identity and the division of physical and mental life emerge early in development, they are not immune to experience and learning. As Marcus Gary (2004) explains, innateness "doesn't mean unmalleable; it means organized in advance of experience" (p. 40). Take, for example, theories of emotions demonstrating that children can experience, express, and identify some "basic emotions" cross-culturally, without learning (Izard 1992). But these emotions are few relative to the expanse of complex emotions that emerge to involve a ref- 
erence to one's self. Self-conscious emotions (i.e., pride, shame, guilt) are a particular type of complex emotion that demonstrate how experience informs children's learning and expression of emotions within their sociocultural environment. Lagattuta and Thompson (2007) review research on these emotions, noting that their emergence requires specific developmental achievements-awareness of self, self-evaluation, social comparison, and even early markers of a theory of mind-but that the roots of these achievements accumulate over time. For example, after 18 months, most children will recognize that a red dot on the forehead of a mirrored reflection is on their forehead and will reach to their own head, not to the reflection (Lewis and Brooks-Gunn 1979), an achievement possible because of many previous experiences of contingent interaction with the world driven what the child is coming to recognize as self. An entirely new repertoire of emotions emerges once children have sufficiently consolidated an intuitive sense of self, especially self as distinct from the external world and self as capable of producing effects. Experience shapes the expression of emotions-and the self that is experiencing them.

Research in developmental psychology on intuitive beliefs represent only a small part of psychological research relevant to discussions of self, identity, and personhood. Another potentially fruitful avenue for advancing conceptions of personhood comes from psychological research on mindfulness. Mindfulness-based interventions teach individuals to evaluate their intuitive sense of self (e.g., the verbal/storied self) against their observing self; to identify thoughts without fusing to them (unquestioningly accepting them as truth, Harris, 2008, p. 33-40). By distinguishing helpful narratives from automatic thoughts, individuals enhance their psychological flexibility rooted in an expanded sense of self.

\section{Neuroscientific Perspectives of Persons}

In contrast to intuitions of a cohesive and coherent singular self, neuroscience research reveals a different conceptualization of personhood: a story of multitudes. Patricia Churchland (2002) argues that neuroscience disabuses the notion "of the self as a singular entity and encourag- 
es the idea that self-representing involves a plurality of functions, each having a range of shades, levels, and degrees" (p. 309). To understand the potential contribution of neuroscience to discussions on personhood, it is important first to understand the basic principles of localized function and distributed processing.

Localized function refers to specialization of specific brain regions in supporting specific functions (Weis et al. 2019). For example, when the New York Times reports that "The more a participant reported feeling passionately in love, the more [the angular gyrus] shifted into overdrive" (Heyman 2017, para. 13), this conveys a research finding for increased activation in the neurons in the angular gyrus, on average, among participants who were in love compared to those less in love and compared to baseline levels of activation in this region. The interpretation, then, is that the brain's processing of romantic love is localized in this brain region. Posner and colleagues (1988) explained localized function by saying that any task, such as the experience of romantic love, "is not performed by any single area of the brain, but the operations that underlie the performance are strictly localized" (p. 1627). This explanation highlights how localized function cannot be understood independently of the principle of distributed processing (Fox and Friston 2012).

Distributed processing of localized functions suggests that any human behavior is not in "spot A," but rather divided into smaller components, each processed across a vast array of interconnected neural networks (Eisenreich, Akaishi, and Hayden 2017). For example, although it is widely accepted that the fusiform face area (FFA) of the brain undergirds facial recognition processing, stressing the activity of the FFA for face processing fails to acknowledge the extent of the integration of brain processing (Fox and Friston 2012) and that "face processing" is a task composed of numerous sub-parts processed in a distributed manner (Sui, Chechlacz, and Humphreys 2012).

Toga and colleagues (2012) suggest that understanding the connections between localized areas of function is better than the more stagnant modular approach to brain functions. They point to the promise of the Human Connectome Project in highlighting the interconnectiv- 
ity and function of specific neural networks that ultimately regulate and produce human activity. The implications of a neural system that is so densely connected-with even more connections within the system (feedback loops) than in connecting the system with the exterior world (feedforward; Bell 1999)-are vast. This is especially true considering the lack of central organizer to make sense of potential conflicts that arise between competing neural networks (Eisenreich et al. 2017).

In this way, modern neuroscience highlights an observation made by Jakob von Uexküll (1934/1992): perception of reality is surely limited by perspective. The umwelt is but a small slice of the reality of the umgebung. David Eagleman (2011) describes the umgebung of the brain as multitudes and teams of rivals, competing to rise to consciousnessthe umwelt. That human brains have constant and inaccessible competition between neural networks can help explain, for example, contradictions between explicit and implicit beliefs and biases. It is possible to hold an explicit belief that conflicts with implicit beliefs about that same topic, when collected under specific experimental conditions. Consider Forstmann and Burgmer's (2015) research on intuitive dualism: participants in their second study demonstrated different patterns of reasoning about body/mind divides when asked explicitly compared to when their reasoning was measured while experiencing cognitive load. In the latter context, it seems likely that different neural networks-normally restricted from consciousness by competing networks-could "slip through" to consciousness because of the experience of cognitive stress, a possibility consistent with other neurobiological explanations of bias (Korteling, Brouwer, and Toet 2018).

This understanding of brain networking, competition, and activation serves as a context to a fundamental question about personhood: how the brain represents the self. Though the literature on the neuroscience of the self is vast, exploring neural substrates that underlie activities such as self-reference, self-concept, self-regulation (e.g., Heatherton 2011; Northoff et al. 2006), one emergent question throughout this literature concerns the extent to which the self is special. Take, for example, the finding that memory is improved when it involves self-reference 
(Symons and Johnson 1997). The neuroscientific question of specialness concerns whether this benefit is because the brain processes self-referent information differently than other kinds of information. Some of the import of this question belies a potential consequence of the lack of specialness; if the self is not special-if it operates like any other construct-then any notion that it is more than an illusory artifact of social processes may be suspect.

Current work on this question is, at this point, insufficient to settle this question though several researchers have suggested that the self is special (Northoff 2016; Sui 2016). Northoff (2016) proposes a model of self that deviates from the commonplace notion that the self is best understood as a higher-order function of the brain. Instead, he suggests that "we need to reverse our model of self; instead of the self being a higherorder cognitive function of either brain or mind, the self may be the most fundamental function of the brain and its intrinsic or spontaneous activity” (p. 204). He ultimately argues that the brain's default network can be understood as overlapping and containing the self, in terms of neural substrates. In other words, the network that is active when there is nothing "happening"-when the brain is at rest-is the network that underlies much of the brain's self-referential processing. Disturbances in this network can cause fundamental disturbances in our sense of self, for example, in the experience of psychosis (Buckner 2013).

Sui (2016) suggests that the self is the "golden thread" in binding, the perceptual process that integrates visual features into a coherent representation of external reality. Moreover, the self seems to play a critical role in the coupling of neural networks (Sui and Humphreys 2015). In contrast to functionalist views of self (e.g., Dennett 2003), Sui and Humphreys argue that because the self is responsible for changing ongoing processes (e.g., in memory, perception), this is consistent with the interpretation of self as mechanism, not as fictional construct. Although persuasive and consistent with the data, Lane, Duncan, Cheng and Northoff (2016) suggest that arguing that the self is special and somehow fundamental does not also require abandoning the conception of the self as a social construction, emerging out of narrative explanations. 
This discussion about the neuroscience of selves does not address theories of extended cognition, which suggests that a robust understanding of the neuroscience of self requires not just drilling down to the multitudes within but also reaching out into the external world of artifacts and relationships inextricably linked to selves (Strawn and Brown 2020). It seems that understanding self as multitudes within and as extended without might open new ways of engaging personhood, with special relevance to Christian doctrines of imago Dei and the Trinity.

\section{Preliminary Conclusions}

Psychological research into personhood thus illuminates a central tension of studying people: there are multiple levels of explanation and they do not necessarily correspond to one another as neatly as one might hope. The methods of psychological science can document what people believe about themselves, how these self-views are influenced by normative developmental processes, and how these views influence important aspects of human functioning across the lifespan. Humans harbor deep-seated intuitions about selves as singular, cohesive, and enduring. Yet, neuroscience research suggests these self-views are myopic. A multitude, rivalry-based network view of personhood-special or not-that is generated by neuroscience is at odds with human intuition. I believe these tensions around intuitive beliefs and neuroscientific explanations about self offer several important starting points for fruitful engagement between empirical sciences, especially psychology and neuroscience, and the humanities, especially philosophy and theology, in understanding personhood.

\section{Implications}

Here I propose three potential contributions to understanding personhood in bridging between science-psychology and neuroscience-and the humanities-philosophy and theology. Specifically, I propose that research in psychology and neuroscience can serve as an anchor for philosophical and theological examinations of the human person, grounding 
those discussions according to evidence about how personhood functions in the lives of people. Additionally, I propose two general ways that psychological thinking about selves can be improved by philosophy/theology: correcting bias and articulating the appropriate boundaries of scientific investigations.

\subsection{Empirical Science Informing Philosophy and Theology}

The preceding discussion highlights a small selection of research on ideas related to self and identity. This research presents a picture that, when zoomed out far enough, is clear: human brains run on de-centralized neural competition and redundancies. These same brains produce a cohesive, continuous, and sturdy sense of self, a self experienced as the "me" that I control. This emergent sense of self develops through experience and relationships (Balswick, King, and Reimer 2016). This suggests an inextricable interconnectedness of the multitudes to the psychological perceptions, and it also highlights how integrating distinct ways of understanding persons is necessary for a phenomenologically thick representation of persons (Franck 2021).

In the same way that Godfrey-Smith (2003) suggests that "Testing in science is typically an attempt to choose between rival hypotheses about the hidden structure of the world" (p. 211), I propose that psychological science about how selves work can provide a useful benchmark for philosophical and theological propositions about selves. The tools available to philosophers and theologians are different than that of a scientist, allowing for theories and arguments that extend beyond empirical data. Yet, when data serve as an anchor to philosophical and theological reasoning, it can provide important feedback on the viability and trustworthiness of specific models, given what the world-and the persons who fill it-are actually like. Scholarly and applied work around concepts of persons, including discussions around a special or a socially constructed self, as well as implications for doctrines of creation (especially the imago Dei) and the doctrine of the Trinity seem especially prepared for this kind of anchoring. One such example of this anchoring comes from Scott Harrower's (2019) work articulating a serious engagement with Trinitarian concepts 
in the context of a scientifically grounded understanding of the effects of trauma and paths toward restoration. In this work, the doctrine of the Trinity is expounded because of its connection to empirical science on lived experience of trauma survivors.

Besides the potential contribution of psychology to the humanities, psychology is also poised to benefit from philosophical and theological thinking around topics of personhood. Although these contributions need not be explicitly limited to research on personhood, this is an area that is particularly qualified to benefit from scrutiny and feedback from non-empirical scholars thinking through these issues.

\subsection{The Humanities Informing Psychology and Neuroscience}

Science is designed to operate as a democratic, self-correcting body of knowledge, yet it is a body of knowledge created by individuals with bias. Ideally, in the long-run, individual bias is overcome by scientific arguments in a public forum between differently biased individuals. When science works, the ideas and explanations that are the most consistent with the evidence and with features of good scientific explanations win out over other ideas (Okasha, 2016). However, bias is psychologically robust (Smith 2020), a fact evident in the social science "replication crisis." Rather than discrediting science, the replication crisis is ultimately good news for scientific progress; it has forced the scientific community to wrestle with diverse types of bias for improved understanding and explanations (Schimmack 2020).

This examination of science by scientists is important because the best explanation for any set of data is not necessarily self-evident. Yet, as Godfrey-Smith (2003) suggests in discussing eliminative inference, "Scientists often think they have ruled out (or rendered very unlikely) all the feasible alternatives to their preferred theory-but in hindsight we can see that in many cases they did not do so, because we now believe a theory they did not even consider" (p. 213). Thus, a first potential contribution to empirical science may come from philosophers and theologians pressing scientists to identify their biases and the assumptions these produce, especially as related to how assumptions influence research questions, pro- 
cesses, and interpretations (Williams 2005). Such an examination may be essential in clarifying the construct itself. Core to the research process is the operational definition of the construct, yet research relevant to discussions of personhood use a wide variety of terms such as self, identity, self-concept, and self-representations. These constructs are inconsistently defined and often lack clarity on how they work together and how they are different (Talaifar and Swann 2018). This difficulty is magnified when neuroscientific explanations of self are included, as this also introduces questions at the center of identity theory (are the neural circuits of self the same thing as self; Myin and Zahnoun, 2018) and consciousness (Blackmore 2005).

A second potential contribution of philosophy and theology to science in the advancement of understanding personhood is in serving as commentators on the proper and necessary boundaries of science (Rosenberg, 2012, p. 1-3). In writing about neuroscience and artificial intelligence (AI), Anthony J. Bell (1999) wrote

Either we are machines, in which case AI should be possible and neuroscience should be able to work out the algorithm (or algorithms) that the brain is running, or we are something else, in which case both projects will fail in their ultimate goals, which is not to say they will not achieve great things along the way. (One of the great things that they might achieve is an exact picture of their own limits.) (p. 2014)

Such a view, I speculate, fails to account for a philosophically informed understanding of the assumptions that underlie science, generally, and neuroscience, specifically (e.g., Hedges and Burchfield, 2005; Slife, Yanchar, and Reber, 2005, p. 4). On the contrary, philosophy can engage the questions that science cannot answer, including why these questions are currently (or permanently) out of science's scope (Rosenberg 2012). Moreover, in questions of normative views (Rosenberg 2012, p. 5-6), theology is especially well-suited to engage psychological science on questions of personhood. In writing about human development, Balswick, King, and Reimer (2016) articulate a fundamental problem in science engaging questions of flourishing and personhood: “developmental theory lacks 
a guiding teleology” (p. 19). Thus, it seems possible that in enhancing and clarifying perspectives, assumptions, and constructs, philosophy can enhance the quality of progress in scientific examinations of personhood. In turn, these enhanced examinations can contribute to theological metanarratives about personhood that are outside scientific inquiry.

\section{Conclusion}

In this paper, I outlined two different empirical ways to explore questions of personhood. Psychological research illuminates developmental constraints of psychological essentialism and intuitive dualism that undergird the subjective experience of a unified self that "I" control. On the other hand, neuroscientific research suggests that the self may not be more than the emergence of some decentralized neural networks over others. In juxtaposing these bodies of research, I suggest empirical data points can inform philosophical and theological models of persons. Likewise, I highlight how philosophical and theological scrutiny of scientific research on selves can inform and enhance research. Ultimately, a clear and robust understanding of personhood will involve bringing together different ways of knowing, methods of investigating, and perspectives within and beyond empirical science.

\section{References}

Ahn, Woo-Kyoung, Caroline C. Proctor, and Elizabeth H. Flanagan. 2009. "Mental Health Clinicians' Beliefs About the Biological, Psychological, and Environmental Bases of Mental Disorders." Cognitive Science 33:147-182. DOI: 10.1111/j.1551-6709.2009.01008.x

Balswick, Jack O., Pamela Ebstyne King, and Kevin S. Reimer. 2016. The Reciprocating Self: Human Development in Theological Perspective. Downers Grove: IVP Academic.

Beaudoin, Cindy, Élizabel Leblanc, Charlotte Gagner, and Miriam H. Beauchamp. 2019. "Systematic Review and Inventory of Theory of Mind Measures for Young Children.” Frontiers in Psychology 10: 2095. DOI: 10.3389/fpsyg.2019.02905 
Bell, Anthony J. 1999. "Levels and Loops: The Future of Artificial Intelligence and Neuroscience." Philosophical Transactions: Biological Sciences 354: 2013-2020. https://www.jstor.org/stable/3030158

Bering, Jesse M. 2006. "The Folk Psychology of Souls." Behavioral and Brain Sciences 29: 453-462. DOI: 10.1017/S0140525X06009101

Blackmore, Susan. 2005. Consciousness: A Very Short Introduction. Oxford: Oxford University Press.

Bloom, Paul. 2004. Descartes' Baby: How the Science of Child Development Explains What Makes Us Human. New York: Basic Books.

Buckner, Randy L. 2013. “The Brain's Default Network: Origins and Implications for the Study of Psychosis.” Dialogues in Clinical Neuroscience 15: 351-358. DOI: 10.31887/DCNS.2013.15.3/rbuckner

Cimpian, Andrei, and Erika Salomon. 2014. "The Inherence Heuristic: An Intuitive Means of Making Sense of the World and a Potential Preursor to Psychological Essentialism." Behavioral and Brain Sciences 37: 461-480. DOI: 10.1017/S0140525X13002197

Chudek, Maciej, Rita Anne McNamara, Susan Birch, Paul Bloon, and Joseph Henrich. 2018. "Do Minds Switch Bodies? Dualist Interpretations Across Ages and Societies.” Religion, Brain, \& Behavior 8:354-368.DOI: 10.1080/2153599X.2017.1377757

Churchland, Patricia. 2002. "Self-Representation in Nervous Systems." Science 296: 308-310. DOI: 10.1126/science1070564

Cohen, Emma, Emily Burdett, Nicola Knight, and Justin Barrett. 2011. "CrossCultural Similarities and Differences in Person-Body Reasoning: Experimental Evidence from the United Kingdom and Brazilian Amazon." Cognitive Science 35:1282-1304. DOI: 10.1111/j.1551-6709.2011.01172.x

Dennett, Daniel C. 2003. "The Self as a Responding-and Responsible-Artifact." Annals of the New York Academy of Sciences 1001: 39-50. DOI: 10.1196/annals.1279.003

Eagleman, David. 2011. Incognito: The Secret Lives of the Brain. New York: Pantheon Books.

Eisenreich, Benjamin R., Rei Akaishi, and Benjamin Y. Hayden. 2017. "Control Without Controllers: Toward a Distributed Neuroscience of Executive Control.” Journal of Cognitive Neuroscience 29: 1684-1698. DOI: 10.1162/jocn_a_01139

Forstmann, Matthias and Pascal Burgmer. 2015. "Adults Are Intuitive Mind-Body Dualists.” Journal of Experimental Psychology: General 144: 222-235. DOI: 10.1037/xge0000045

Forstmann, Matthias, Pascal Burgmer, and Thomas Mussweiler. 2012. " 'The Mind Is Willing, but the Flesh Is Weak': The Effects of Mind-Body Du- 
alism on Health Behavior.” Psychological Science 23: 1239-1245. DOI: 10.1177/0956797612442392

Fox, Peter T., and Karl J. Friston. 2012. “Distributed Processing; Distributed Functions?” NeuroImage 61: 407-426. DOI: 10.1016/j.neuroimage.2011.12.051 Franck, Juan F. 2021. “The Person at the Core of Psychological Science.” Scientia et Fides 9 (2): 15-33. DOI: 10.12775/SetF.2021.016.

Gary, Marcus. 2004. The Birth of the Mind: How a Tiny Number of Genes Creates the Complexities of Human Thought. New York: Basic Books.

Gelman, Susan A. 2003. The Essential Child: Origins of Essentialism in Everyday Thought. Oxford: Oxford University Press.

Gelman, Susan A., and Henry M. Wellman. 1991. "Insides and Essence: Early Understandings of the Non-Obvious.” Cognition 38: 213-244. DOI: 10.1016/00100277(91)9007-Q

Godfrey-Smith, Peter. 2003. Theory and Reality: An Introduction to the Philosophy of Science. Chicago: The University of Chicago Press.

Harris, Russ. 2008. The Happiness Trap: How to Stop Struggling and Start Living. Boulder: Trumpeter.

Harrower, Scott. 2019. God of All Comfort: A Trinitarian Response to the Horrors of This World. Bellingham: Lexham Press.

Heatherton, Todd F. 2011. "Neuroscience of Self and Self-Regulation.” Annual Review of Psychology 62: 363-390. DOI: 10.1146/annurev.psych.121208.131616

Hedges, Dawson, and Colin Burchfield. 2005. "The Assumptions and Implications of the Neurobiological Approach to Depression.” In Critical Thinking about Psychology: Hidden Assumptions and Plausible Alternatives, edited by Brent D. Slife, Jeffrey S. Reber, and Frank C. Richardson, 99-120. Washington DC: American Psychological Association.

Heyman, Stephen. 2017, November 8. “Don’t Know What the Angular Gyrus Is? Your Heart Does.” The New York Times. https://www.nytimes.com/2017/11/08/ style/modern-love-neuroscience.html

Heyman, Gail D. and Jessica W. Giles. 2006. “Gender and Psychological Essentialism.” Enfance 58: 293-310. https://www.ncbi.nlm.nih.gov/pmc/articles/ PMC3082140/

Hodge, K. Mitch. 2008. “Descartes’ Mistake: How Afterlife Beliefs Challenge the Assumption that Humans are Intuitive Cartesian Substance Dualists.” Journal of Cognition and Culture 8: 387-415. DOI: 10.1163/156853708X358236

Hood, Bruce, Nathalia L. Gjersoe, and Paul Bloom. 2012. "Do Children Think that Duplicating the Body Also Duplicates the Mind?" Cognition 125: 466-474. DOI: 10.1016/j.cognition.2012.07.005 
Izard, Carroll E. 1992. "Basic Emotions, Relations Among Emotions, and EmotionCognition Relations.” Psychological Review 99: 561-565. DOI: 10.1037/0033295X.99.3.561

Johnson, Carl Nils. 1990. "If You Had My Brain, Where Would I Be? Children’s Understanding of the Brain and Identity." Child Development 61: 962-972. DOI: $10.2307 / 1130868$

Keil, Frank C. 1992. “The Origins of an Autonomous Biology.” In The Minnesota Symposia on Child Psychology, Vol. 25, Modularity and Constraints in Language and Cognition, edited by Megan R. Gunnar and Michael Maratsos, 103-137. Hilldale: Lawrence Erlbaum Associates, Inc.

Korteling, Johan E., Anne-Marie Brouwer, and Alexander Toet. 2018. "A Neural Network Framework for Cognitive Bias.” Frontiers in Psychology 9: 1561. DOI: 10.3389/fpsyg.2018.01561

Lagattuta, Kristin Hansen, and Ross A. Thompson. 2007. "The Development of Self-Conscious Emotions: Cognitive Processes and Social Influences." In The Self-Conscious Emotions: Theory and Research, edited by Jessica L. Tracy, Richard W. Robins, and June Price Tangney, 91-113. New York: The Guilford Press.

Lane, Timothy, Niall W. Duncan, Tony Cheng, and Georg Northoff. 2016. "The Trajectory of Self.” Trends in Cognitive Sciences 20: 481-482. DOI: 10.1016/j. tics.2016.03.004

Lewis, Michael, and Jeanne Brooks-Gunn. 1979. Social Cognition and the Acquisition of Self. New York: Plenum Press.

Myin, Erik and Farid Zahnoun. 2018. "Reincarnating the Identity Theory." Frontiers in Psychology 9: 2044. DOI: 10.3389/fpsyg.2018.02044

Newman, George E., and Frank C. Keil. 2008. "Where Is the Essence? Developmental Shifts in Children's Beliefs About Internal Features.” Child Development 79: 1344-1356. DOI: 10.1111/j.1467-8624.2008.01192.x

Northoff, Georg. 2016. "Is the Self a Higher-Order or Fundamental Function of the Brain? The 'Basis Model of Self-Specificity' and its Encoding by the Brain's Spontaneous Activity.” Cognitive Neuroscience 7: 1-4. DOI: 10.1080/17588928.2015.1111868

Northoff, Georg, Alexander Heinzel, Moritz de Greck, Felix Bermpohl, Henrik Dobrowolny, and Jaak Panksepp. 2006. "Self-Referential Processing In Our Brain-A Meta-Analysis of Imaging Studies on the Self.” NeuroImage 31: 440457. DOI: 10.1016/j.neuroimage.2005.12.002

Okasha, Samir. 2016. Philosophy of Science: A Very Short Introduction. Oxford: Oxford University Press. 
Posner, Michael I., Steven E. Peterson, Peter T. Fox, and Marcus E. Richle. 1988. "Localization of Cognitive Operations in the Human Brain." Science 240: 1627-1631. https://www.jstor.org/stable/1701013

Premack, David, and Guy Woodruff. 1978. “Does the Chimpanzee Have a Theory of Mind?" The Behavioral and Brain Sciences 4: 515-526. DOI: 10.1017/ S0140525X00076512

Richert, Rebekah A., and Paul L. Harris 2008. “Dualism Revisited: Body vs. Mind vs. Soul.” Journal of Cognition and Culture 8: 99-115. DOI: 10.1163/156770908 X289224

Riekki, Tapani, Marjaana Lindeman, and Jari Lipsanen. 2013. “Conceptions about the mind-body problem and their relations to afterlife beliefs, paranormal beliefs, religiosity, and ontological confusions." Advances in Cognitive Psychology 9: 112-120. DOI: 10.2478/v10053-008-0138-5

Roazzi, Maira, Melanie Nyhof, and Carl Johnson. 2013. "Mind, Soul, and Spirit: Conceptions of Immaterial in Different Cultures." International Journal for the Psychology of Religion 23: 75-86. DOI: 10.1080/10508619.2013.735504

Rosenberg, Alex. 2012. Philosophy of Science: A Contemporary Introduction ( ${ }^{\text {rd }}$ Edition). New York: Routledge.

Schimmack, Ulrich. 2020. “A Meta-Psychological Perspective on the Decade of Replication Failures in Social Psychology.” Canadian Psychology/Psychologie Canadienne 61: 364-376. DOI: 10.1037/cap0000246

Slife, Brent D., Stephen C. Yanchar, and Jeffrey S. Reber. 2005. "Introduction: Thinking Critically About Critical Thinking." In Critical Thinking about Psychology: Hidden Assumptions and Plausible Alternatives, edited by Brent D. Slife, Jeffrey S. Reber, and Frank C. Richardson, 3-14. Washington DC: American Psychological Association.

Smith, Erin I. 2020. “The Role of Psychology in Advancing Dialogue Between Science and Christianity.” Perspectives on Science and Christian Faith 71: 204-221.

Strawn, Brad D., and Warren S. Brown. 2020. Enhancing Christian Life: How Extended Cognition Augments Religious Community. Downers Grove: IVP Academic.

Sui, Jie. 2016. "Self-Reference Acts as a Golden Thread in Binding." Trends in Cognitive Sciences 20: 482-483. DOI: 10.1016/j.yics.2016.04.005

Sui, Jie, Magdalena Chechlacz, and Glyn W. Humphreys. 2012. “Dividing the Self: Distinct Neural Substrates of Task-Based and Automatic Self-Prioritization After Brain Damage.” Cognition 122: 150-162. DOI: 10.1016/j.cognition.2011.10.008 
Sui, Jei, and Glyn W. Humphreys. 2015. "The Integrative Self: How Self-Reference Integrates Perception and Memory.” Trends in Cognitive Sciences 19: 719-728. DOI: 10/1016/j.tics.2015.08.015

Symons, Cynthia S., and Blair T. Johnson. 1997. "The Self-Reference Effect in Memory: A Meta-Analysis.” Psychological Bulletin 121: 371-394. DOI: 10.1037/0033-2909.121.3.371

Talaifar, Sanaz, and Willaim Swann. 2018. "Self and Identity.” In Oxford Research Encyclopedia of Psychology. DOI: 10.1093/acrefore/9780190236557.013.242

Toga, Arthur W., Kristi A. Clark, Paul M. Thompson, David W. Shattuck, and John Darrell Van Horn. 2012. "Mapping the Human Connectome." Neurosurgery 71: 1-5. DOI: 10.1227/NEU.0b013e318258e9ff

Von Uexküll, Jakob. 1934/1992. "A Stroll Through the Worlds of Animals and Men: A Picture Book of Invisible Worlds.” 89: 319-391. DOI: 10.1515/ semi.1992.89.4.319

Weis, Serge, Michael Sonnberger, Andreas Dunzinger, Eva Voglmayr, Martin Aichholzer, Raimund Kleiser, and Peter Strasser. 2019. "Localization of Brain Function.” In Imaging Brain Diseases, 401-423. Vienna: Springer. DOI: 10.1007/978-3-7091-1544-2_14

Williams, Richard N. 2005. "The Language and Methods of Science: Common Assumptions and Uncommon Conclusions." In Critical Thinking about Psychology: Hidden Assumptions and Plausible Alternatives, edited by Brent D. Slife, Jeffrey S. Reber, and Frank C. Richardson, 235-249. Washington DC: American Psychological Association. 